\title{
Phytochemical Composition of Tridax procumbens Linn Leaves: Potential as a Functional Food
}

\author{
Catherine C. Ikewuchi, Jude C. Ikewuchi*, Mercy O. Ifeanacho \\ Department of Biochemistry, Faculty of Biological and Chemical Sciences, College of Natural and Applied \\ Sciences, University of Port Harcourt, Port Harcourt, Nigeria \\ Email: ${ }^{*}$ ecoli240733@yahoo.com
}

Received 21 February 2015; accepted 12 August 2015; published 17 August 2015

Copyright (C) 2015 by authors and Scientific Research Publishing Inc.

This work is licensed under the Creative Commons Attribution International License (CC BY). http://creativecommons.org/licenses/by/4.0/

(c) (i) Open Access

\section{Abstract}

The leaves of Tridax procumbens were screened for the presence of bioactive molecules. They had high flavonoids, alkaloids, hydroxycinnamates, tannins and phytosterols, moderate benzoic acid derivatives and lignans, and low carotenoids contents. Thirty nine known alkaloids (mainly akuammidine, 68.756\%), twenty three known flavonoids (mainly $17.593 \%$ kaempferol and $\mathbf{1 2 . 5 3 8 \%}(-)$-epicatechin), five known carotenoids (mainly lutein, $62.608 \%$ ), four known benzoic acid derivatives (mainly ferulic acid, $46.091 \%$ ), two phytosterols (mainly stigmasterol, $80.853 \%$ ) and six known lignans (mainly galgravin, $77.326 \%$ ) were detected. Also detected were caffeic acid and tannic acid. The medicinal properties of the flavonoids, phytosterols, alkaloids, tannins, hydroxicinnamates, carotenoids, benzoic acid derivatives and lignans that were present in the leaves were discussed herein and proposed to be explored for their potential medicinal values. The great number of potentially active nutrients and their multifunctional properties make Tridax procumbens a perfect candidate for the production of health-promoting food and food supplements.

\section{Keywords}

Flavonoids, Hydroxycinnmates, Lignans, Nutraceuticals, Phytosterol, Tannins

\section{Introduction}

Natural products and health foods have recently received a lot of attention both by health professionals and the common population for improving overall well-being, as well as in the prevention of diseases. Epidemiological

"Corresponding author.

How to cite this paper: Ikewuchi, C.C., Ikewuchi, J.C. and Ifeanacho, M.O. (2015) Phytochemical Composition of Tridax procumbens Linn Leaves: Potential as a Functional Food. Food and Nutrition Sciences, 6, 992-1004. 
evidence has associated the frequent consumption of vegetables with decreased disease risk [1]-[3]. In line with this, different types of vegetables have been re-evaluated for recognition for possible candidature as functional foods, and as valuable sources of nutraceuticals. Functional foods refer to those whole, fortified, enriched or enhanced foods that provide health benefits beyond the provision of essential nutrients (e.g., vitamins and minerals), when they are consumed at efficacious levels as part of a varied diet on a regular basis [1] [4]. The term nutraceutical, which combines the word nutrition and pharmaceutical, is defined as a food that contains a medical health benefit beyond that of basic nutrition [1] [2] [4].

One such vegetables with a potential for use as functional food is Tridax procumbens Linn (family Asteraceae), which is commonly called coat buttons. Its leaves are cooked and eaten as vegetable, and are also used as fodders [5]-[8]. Consequent upon this, Ikewuchi and colleagues investigated the nutritional potential of the leaves [9]-[11]. The leaves are also commonly used for medicinal purposes, because of their myriad of pharmacological properties. These include analgesic [12], anti-anemic [13], anti-arthritic [14], anti-diabetic [15]-[17], antihypertensive [18] [19], anti-inflammatory, antioxidant [20], antimicrobial [21], antipyretic [6], hepatoprotective [22], hypocholesterolemic and weight reducing [23] properties. The present study reports the phytochemical composition of the leaves of Tridax procumbens, and in addition discusses the bioactivities of the detected compounds, with a view to highlighting the possibilities of the use of the leaves as a functional food, or as a source of nutraceuticals.

\section{Materials and Methods}

\subsection{Collection of Plant Samples}

Samples of fresh Tridax procumbens plants were collected from within the Choba and Abuja Campuses of University of Port Harcourt, Nigeria. Their identity was confirmed by Dr. M.C. Dike of Taxonomy Unit, Department of Forestry and Environmental Management, Michael Okpara University of Agriculture, Umudike, Abia State, Nigeria; and Mr. John Ibe, the Herbarium Manager of the Forestry Department, National Root Crops Research Institute, Umuahia, Nigeria. They were cleaned and their leaves were removed, oven dried at $55^{\circ} \mathrm{C}$ and grounded into powder, which was stored in an air tight container, for subsequent use.

\subsection{Determination of the Phytochemical Content of the Leaves}

\subsubsection{General Experimental Procedures}

Analyses were carried out at Multi Environmental Management Consultants Limited, Igbe Road, Ikorodu, Lagos, Nigeria, with a Hewlett Packard HP 6890, GC apparatus, fitted with flame ionization detector, powered with HP Chemstation Rev. A 09.01 (1206) software, to identify and quantify compounds. Standard solutions were prepared in methanol for alkaloids, flavonoids, tannins and simple benzoic acid derivatives; acetone for carotenoids and lignans, methylene chloride for phytosterols; and ethanol for hydroxycinnamates. The linearity of the dependence of response on concentration was verified by regression analysis. Identification was based on comparison of retention times and spectral data with standards. Quantification was performed by establishing calibration curves for each compound determined, using the standards.

\subsubsection{Determination of Alkaloid Composition}

Extraction was carried out according to the method of Tram et al. [24]. The extract was subjected to gas chromatography on a capillary DB-5MS column $(30 \mathrm{~m} \times 0.25 \mathrm{~mm} \times 0.25 \mu \mathrm{m}$ film thickness). The inlet and detection temperatures were $250^{\circ} \mathrm{C}$ and $320^{\circ} \mathrm{C}$. Split injection (split ratio of 20:1) was adopted. The carrier gas was nitrogen. The hydrogen and compressed air pressures were 1.97 and $2.67 \mathrm{~kg} / \mathrm{cm}^{2}$. The oven was programmed initially at $60^{\circ} \mathrm{C}$ for $5 \mathrm{~min}$, ramped at $10^{\circ} \mathrm{C} / \mathrm{min}$ for $20 \mathrm{~min}$, before ramping again at $15^{\circ} \mathrm{C} / \mathrm{min}$ for $4 \mathrm{~min}$.

\subsubsection{Determination of Carotenoid Composition}

The extraction was carried out by a modification of the method of Rodriguez-Amaya and Kimura [25]. The carotenoids were successively extracted with acetone and a (1:1) mixture of diethyl ether and petroleum ether, were concentrated and re-dissolved in acetone before saponifying and re-extracting with a (1:1) mixture of diethyl ether and petroleum ether. The resultant extracts were dried and re-dissolved in petroleum ether and subjected to gas chromatography on a capillary ZP-5 column (30 $\mathrm{m} \times 0.32 \mathrm{~mm} \times 0.25 \mu \mathrm{m}$ film thickness). The oven 
was programmed initially at $45^{\circ} \mathrm{C}$, held for $6 \mathrm{~min}$, and ramped at $38^{\circ} \mathrm{C} / \mathrm{min}$ to $250^{\circ} \mathrm{C}$. Initial column head pressure at a constant flow mode was $0.243 \mathrm{~kg} / \mathrm{cm}^{2}$.

\subsubsection{Determination of Flavonoid Composition}

The extraction was carried out according to the method of Millogo-Kone et al. [26]. The extract was subjected to gas chromatography on a capillary HP INNOWax column $(30 \mathrm{~m} \times 0.25 \mathrm{~mm} \times 0.25 \mu \mathrm{m}$ film thickness). The inlet and detection temperatures were $250^{\circ} \mathrm{C}$ and $320^{\circ} \mathrm{C}$. Split injection (split ratio of 20:1) was adopted. The carrier gas was nitrogen. The hydrogen and compressed air pressures were 1.55 and $2.46 \mathrm{~kg} / \mathrm{cm}^{2}$. The oven was programmed initially at $50^{\circ} \mathrm{C}$, ramped at $8^{\circ} \mathrm{C} / \mathrm{min}$ for $20 \mathrm{~min}$, maintained for $4 \mathrm{~min}$, ramped again at $12^{\circ} \mathrm{C} / \mathrm{min}$ for 4 min, and maintained for 4 min.

\subsubsection{Determination of Hydroxycinnamates Composition}

The extraction was carried according to method of Ortan et al. [27]. The extract was subjected to gas chromatography on HP-5 column (30 $\mathrm{m} \times 0.32 \mathrm{~mm} \times 0.25 \mu \mathrm{m}$ film thickness). It was introduced via an all-glass injector working in the split mode, with nitrogen as the carrier gas, at a flow rate of $1 \mathrm{~mL} / \mathrm{min}$. The injection and detector temperatures were $260^{\circ} \mathrm{C}$ and $300^{\circ} \mathrm{C}$. The oven was programmed at the start of the run from $170^{\circ} \mathrm{C}$ to $250^{\circ} \mathrm{C}$ at $5^{\circ} \mathrm{C} / \mathrm{min}$.

\subsubsection{Determination of the Lignans Composition}

The extraction was carried out according to the method of Chapman et al. [28]. The extract was subjected to gas chromatography on a ZP-5 column ( $30 \mathrm{~m} \times 0.32 \mathrm{~mm} \times 0.25 \mu \mathrm{m}$ film thickness). One microliter of sample was injected. The initial oven temperature was $40^{\circ} \mathrm{C}$, while the injector and transfer line temperatures were $250^{\circ} \mathrm{C}$ and $280^{\circ} \mathrm{C}$. A solvent delay of $2.00 \mathrm{~min}$ was followed by ramping at $10^{\circ} \mathrm{C} / \mathrm{min}$ to a final temperature of $230^{\circ} \mathrm{C}$ and held for $1.00 \mathrm{~min}$.

\subsubsection{Determination of Benzoic Acid Derivatives Composition}

The extraction was carried out according to the method of Ndoumou et al. [29]. The extract was subjected to gas chromatography on a capillary HP 1 column $(30 \mathrm{~m} \times 0.25 \mathrm{~mm} \times 0.25 \mu \mathrm{m}$ film thickness $)$. The inlet and detection temperatures were $250^{\circ} \mathrm{C}$ and $320^{\circ} \mathrm{C}$. Split injection (split ratio of 20:1) was adopted. The carrier gas was nitrogen, at a pressure of $2.11 \mathrm{~kg} / \mathrm{cm}^{2}$. The hydrogen and compressed air pressures were 1.97 and $2.25 \mathrm{~kg} / \mathrm{cm}^{2}$. The oven was programmed initially at $60^{\circ} \mathrm{C}$ for $5 \mathrm{~min}$, ramped at $15^{\circ} \mathrm{C} / \mathrm{min}$ for $15 \mathrm{~min}$, maintained for $1 \mathrm{~min}$, and then ramped again at $10^{\circ} \mathrm{C} / \mathrm{min}$ for $4 \mathrm{~min}$.

\subsubsection{Determination of Phytosterols Composition}

Extraction of oil was carried out according to AOAC method 999.02 [30], while the analysis of sterols was carried out according to AOAC method 994.10 [30]. The resultant sterol derivatives were subjected to gas chromatography on a capillary HP INNOWax column ( $30 \mathrm{~m} \times 0.25 \mathrm{~mm} \times 0.25 \mu \mathrm{m}$ film thickness). The inlet and detection temperatures were $250^{\circ} \mathrm{C}$ and $320^{\circ} \mathrm{C}$. Split injection (split ratio of 20:1) was adopted. The carrier gas was nitrogen. The hydrogen and compressed air pressures were 1.55 and $2.46 \mathrm{~kg} / \mathrm{cm}^{2}$. The oven was programmed initially at $60^{\circ} \mathrm{C}$, ramped at $10^{\circ} \mathrm{C} / \mathrm{min}$ for $20 \mathrm{~min}$, maintained for $4 \mathrm{~min}$, ramped again $15^{\circ} \mathrm{C} / \mathrm{min}$ for $4 \mathrm{~min}$, and maintained for $10 \mathrm{~min}$.

\subsubsection{Determination of Tannins Composition}

Extraction was carried out according to the method of Luthar [31]. The extract was subjected to gas chromatography on HP 5 column ( $30 \mathrm{~m} \times 0.25 \mathrm{~mm} \times 0.25 \mu \mathrm{m}$ film thickness). The inlet and detection temperatures were $250^{\circ} \mathrm{C}$ and $320^{\circ} \mathrm{C}$. Split injection (split ratio of 20:1) was adopted. The carrier gas was nitrogen. The hydrogen and compressed air pressures were 1.97 and $2.81 \mathrm{~kg} / \mathrm{cm}^{2}$. The oven was programmed initially at $120^{\circ} \mathrm{C}$, before ramping at $10^{\circ} \mathrm{C} / \mathrm{min}$ for $20 \mathrm{~min}$.

\subsection{Derivation of Composition per Wet Weight from the Composition per Dry Weight}

The compositions per wet weight of the determined parameters were derived from the compositions per dry weight and vice versa, using the following formula, adapted from Health Canada Official Methods [32]. 


$$
\text { Composition per wet weight }(\%)=\frac{\text { composition per dry weight }(\%) \times \text { dry matter content }(\%)}{100}
$$

\subsection{Data Analysis}

Comparisons were based on simple percentages.

\section{Results}

The leaves had high flavonoids content $(6477.706 \mathrm{~g} / \mathrm{kg})$ (Table 1). Twenty three known flavonoids were detected in the leaves. This consisted of 17.593\% kaempferol, 12.538\% (-)-epicatechin, 8.202\% biochanin, $7.968 \%$ (+)-catechin, $7.875 \%$ apigenin, $6.254 \%$ naringenin, $6.124 \%$ daidzein, $4.418 \%$ quercetin, $4.376 \%$ butein, 3.895\% robinetin, 3.047\% baicalein, $2.986 \%$ nobiletin, $2.806 \%$ (-)-epigallocatechin, $2.444 \%$ genistein, $2.338 \%(+)-$ gallocatechin, $1.818 \%$ ellagic acid, $1.707 \%$ luteolin, $1.406 \%$ myricetin, $0.735 \%$ baicalin, $0.498 \%$ isorhamnetin, 0.404\% (-)-epigallocatechin-3-gallate, 0.359\% silymarin and 0.207\% (-)-epicatechin-3-gallate.

Table 1. Flavonoids composition of Tridax procumbens leaves.

\begin{tabular}{|c|c|c|c|}
\hline \multirow{2}{*}{ Compounds } & \multirow{2}{*}{$\begin{array}{l}\text { Retention } \\
\text { time (min) }\end{array}$} & \multicolumn{2}{|c|}{ Composition (mg/kg) } \\
\hline & & /dry weight & /wet weight \\
\hline$(+)$-Catechin & 13.749 & 5187.515 & 516.158 \\
\hline (+)-Gallocatechin & 15.043 & 1522.281 & 151.467 \\
\hline Genistein & 15.626 & 1590.816 & 158.286 \\
\hline Daidzein & 16.047 & 3986.855 & 396.692 \\
\hline Apigenin & 16.380 & 5127.089 & 510.145 \\
\hline Butein & 16.680 & 2849.149 & 283.490 \\
\hline Naringenin & 16.792 & 4071.731 & 405.137 \\
\hline Biochanin & 17.101 & 5339.931 & 531.323 \\
\hline Luteolin & 17.371 & 1111.266 & 110.571 \\
\hline Kaempferol & 18.503 & $11,453.533$ & 1139.627 \\
\hline (-)-Epicatechin & 19.530 & 8162.805 & 812.199 \\
\hline (-)-Epigallocatechin & 20.478 & 1826.631 & 181.750 \\
\hline Quercetin & 21.445 & 2875.972 & 286.159 \\
\hline (-)-Epicatechin-3-gallate & 22.610 & 134.977 & 13.430 \\
\hline (-)-Epigallocatechin-3-gallate & 23.238 & 263.286 & 26.197 \\
\hline Isorhamnetin & 24.104 & 324.132 & 32.251 \\
\hline Robinetin & 24.193 & 2535.979 & 252.330 \\
\hline Ellagic acid & 24.546 & 1183.313 & 117.740 \\
\hline Myricetin & 24.796 & 915.467 & 91.089 \\
\hline Baicalein & 25.701 & 1983.819 & 197.390 \\
\hline Nobiletin & 26.356 & 1944.091 & 193.437 \\
\hline Baicalin & 26.956 & 478.418 & 47.603 \\
\hline Silymarin & 27.813 & 233.524 & 23.236 \\
\hline Total flavonoids & - & $65,102.577$ & 6477.706 \\
\hline
\end{tabular}


The leaves had high hydroxycinnamates $(0.580 \mathrm{~g} / \mathrm{kg})$, tannins $(0.813 \mathrm{~g} / \mathrm{kg})$ and phytosterols $(0.250 \mathrm{~g} / \mathrm{kg})$, moderate benzoic acid derivatives $(0.032 \mathrm{~g} / \mathrm{kg})$ and lignans $(0.077 \mathrm{~g} / \mathrm{kg})$, and low carotenoids $(0.001 \mathrm{~g} / \mathrm{kg})$ contents (Table 2). Five known carotenoids were detected in the leaves. This consisted of $62.608 \%$ lutein, $13.989 \%$ carotene, $11.328 \%$ antheraxanthin, $8.532 \%$ neoxanthin and 3.542\% violaxanthin. Four known benzoic acid derivatives were detected in the leaves. This consisted of $46.091 \%$ ferulic acid, $22.624 \%$ 4-hydroxybenzaldehyde, $16.441 \%$ vanillic acid and $14.843 \%$ 4-hydroxybenzoic acid. The hydroxycinnamates fraction consisted $100 \%$ of caffeic acid, the tannins fraction consisted $100 \%$ of tannic acid, while the phytosterols fraction consisted of stigmasterol (80.853\%) and sitosterol (19.146\%). The leaves had moderate lignans $(0.077 \mathrm{~g} / \mathrm{kg})$ contents (Table $3)$. Six known lignans were detected in the leaves. This consisted of $77.326 \%$ galgravin, $12.221 \%$ dehydroabietic acid, $7.837 \%$ retusin, $2.612 \%$ epieudesmin, $0.003 \%$ apigenin-4', 7-dimethyl ether and $0.00002 \%$ (9E,12E, 15E)-9,12,15-octadecatrien-1-ol.

Table 2. Benzoic acid derivatives, carotenoids, phytosterols, hydroxycinnamates and tannins compositions of Tridax procumbens leaves.

\begin{tabular}{|c|c|c|c|}
\hline \multirow{2}{*}{ Compounds } & \multirow{2}{*}{$\begin{array}{l}\text { Retention } \\
\text { time (min) }\end{array}$} & \multicolumn{2}{|c|}{ Composition (mg/kg) } \\
\hline & & /dry weight & /wet weight \\
\hline \multicolumn{4}{|l|}{ Benzoic acid derivatives } \\
\hline 4-Hydroxybenzaldehyde & 8.947 & 73.708 & 7.334 \\
\hline 4-Hydroxybenzoic acid & 12.363 & 48.358 & 4.812 \\
\hline Vanillic acid & 15.234 & 53.564 & 5.330 \\
\hline Ferulic acid & 18.052 & 150.161 & 14.941 \\
\hline Total benzoic acid derivatives & & 325.792 & 32.416 \\
\hline \multicolumn{4}{|l|}{ Carotenoids } \\
\hline Neoxanthin & 19.525 & 1.2112 & 0.1205 \\
\hline Viola-xanthin & 20.542 & 0.5028 & 0.0500 \\
\hline Anthera-xanthin & 21.441 & 1.6080 & 0.1600 \\
\hline Carotene & 23.191 & 1.9856 & 0.1980 \\
\hline Lutein & 24.796 & 8.8870 & 0.8843 \\
\hline Total carotenoids & & 14.1947 & 1.4124 \\
\hline \multicolumn{4}{|l|}{ Phytosterols } \\
\hline Stigmasterol & 23.264 & 2032.970 & 202.281 \\
\hline Sitosterol & 24.802 & 481.414 & 47.901 \\
\hline Total phytosterols & & 2514.390 & 250.182 \\
\hline \multicolumn{4}{|l|}{ Tannins } \\
\hline Tannic acid & 19.223 & 8175.408 & 813.453 \\
\hline Total tannins & & 8175.408 & 813.453 \\
\hline \multicolumn{4}{|l|}{ Hydroxycinnamates } \\
\hline Caffeic acid & 14.096 & 5830.871 & 580.172 \\
\hline Total hydroxycinnamates & & 5830.871 & 580.172 \\
\hline
\end{tabular}


Table 3. Lignans composition of Tridax procumbens leaves.

\begin{tabular}{cccc}
\hline \multirow{2}{*}{ Compounds } & Retention & \multicolumn{2}{c}{ Composition (mg/kg) } \\
\cline { 4 - 4 } & time (min) & /dry weight & /wet weight \\
\hline (9E,12E,15E)-9,12,15-Octadecatrien-1-ol & 14.095 & 0.00014 & $0.000,01$ \\
Apigenin-4',7-dimethyl ether & 16.270 & 0.02540 & $0.002,53$ \\
Dehydroabietic acid & 18.618 & $94.933,90$ & $9.445,92$ \\
Retusin & 19.625 & $60.875,60$ & $6.057,12$ \\
Galgravin & 20.444 & $600.666,70$ & $59.766,34$ \\
Epieudesmin & 22.324 & $20.293,10$ & $2.019,16$ \\
Total lignans & & $776.794,70$ & $77.291,07$ \\
\hline
\end{tabular}

The leaves had very high alkaloids content (10.191 g/kg) (Table 4). Thirty nine known alkaloids were detected in the leaves. This consisted of $68.756 \%$ akuammidine, $16.508 \%$ voacangine, $4.944 \%$ echitamine, $2.3167 \%$ lupanine, $2.253 \%$ angustifoline, $1.902 \%$ augustamine, $0.549 \%$ crinamidine, $0.477 \%$ echitamidine, $0.327 \%$ cinchonidine, $0.324 \%$ oxoassoanine, $0.317 \%$ trigonelline, $0.178 \%$ crinane- $3 \alpha$-ol, $0.177 \%$ choline, $0.141 \%$ cinchonine, $0.117 \%$ indicine-N-oxide, $0.093 \%$ monocrotaline, $0.079 \%$ nitidine, $0.064 \%$ 6-hydroxypowelline, $0.050 \%$ 13- $\alpha$-hydrorhombifoline, $0.043 \%$ acronycine, $0.041 \%$ powelline, $0.041 \%$ ambelline, $0.040 \% 6$-hydroxyundulatine, $0.040 \%$ dihydro-oxo-demethoxyhaemanthamine, $0.039 \%$ 9-octadecenamide, $0.037 \%$ ellipcine, $0.030 \%$ 1 $\beta, 2 \beta$-epoxyambelline, $0.028 \%$ buphanidrine, $0.026 \%$ undulatine, $0.020 \%$ 6-hydroxybuphanidrine, $0.016 \%$ sparteine, etc.

\section{Discussion}

The leaves had higher catechin, gallocatechin, epicatechin, epigallocatechin, epicatechin gallate, epigallocatechin gallate, naringenin, apigenin, luteolin, kaempferol, myricetin and quercetin contents than lettuce and onions [33]. Amongst the biological activities of flavonoids are actions against free radicals, free radical mediated cellular signaling, inflammation, allergies, platelet aggregation, microbes, ulcers, viruses, tumors and hepatotoxins [34]. Luteolin has antibacterial, anti-inflammatory, anti-mutagenic, antioxidant [34], anti-allergic, anti-androgenic, anticancer, anti-diabetic, anti-estrogenic, antimicrobial, hypocholesterolemic, hypotensive, neuroprotective and radio-protective [35] activities. Kaempferol is known for its antibacterial, anti-inflammatory, antioxidant [34], analgesic, anti-allergic, anticancer, anti-diabetic, antifungal, antiprotozoal, anti-osteoporotic, antithrombogenic, antiviral, cardioprotective, hepatoprotective, hypocholesterolemic, hypotriglyceridemic and neuroprotective [36] activities. Kaempferol-3-O-rutinoside has hypotensive activity [37].

Apigenin has antibacterial, anti-inflammatory, diuretic, hypotensive, smooth muscle relaxation enhancing [34], antioxidant, anti-carcinogenic, antidepressant, cardioprotective, hepatoprotective, anti-peroxidative [38], anti-tumor, anti-proliferative, oxygenase inhibitory and apoptosis inducing [39] activities. Naringenin has antiatherogenic, anticancer, anti-fibrogenic, anti-inflammatory, anti-mutagenic, antioxidant, hepatoprotective, hypocholesterolemic [40], anti-ulcer and cardioprotective [41] properties.

Catechins are antimicrobial [34], anti-allergic, anti-carcinogenic, anti-diabetic, antihypertensive, anti-inflammatory, anti-mutagenic, anti-obesity, antioxidant, anti-platelet, anti-proliferative, anti-tumorigenic, antiulcer, chemo-preventive, hypocholesterolemic and neuroprotective [42] agents. (+)-Catechin has been reported to have cardioprotective [34], antispasmodic, bronchodilator and vasodilator [43] activities. Epicatechin, epicatechin gallate and epigallocatechin gallate provide protective benefits by their free radical scavenging ability and their inhibition of eicosanoid synthesis and platelet aggregation [34]. In addition, epicatechin has antioxidant [34] and hypoglycemic activities [44].

Diadzein and genistein have antithrombotic, hypocholesterolemic, hypotensive [45], anticancer and estrogenlike properties [34]. Genistein also has anti-diabetic, anti-inflammatory, anti-obesity, antioxidant and cardioprotective [46] activities. Nobiletin has anti-atherosclerotic, anti-dyslipidemic, antihypertensive, anti-inflammatory, antioxidant, anti-resorptive, antithrombotic [47], anti-angiogenic, anti-carcinogenic, anti-dementia, cognitive impairment improving [48] and antitumor properties, and enhances adiponectin secretion [49]. 
Table 4. Alkaloids composition of Tridax procumbens leaves.

\begin{tabular}{|c|c|c|c|}
\hline \multirow{2}{*}{ Compounds } & \multirow{2}{*}{$\begin{array}{l}\text { Retention } \\
\text { time (min) }\end{array}$} & \multicolumn{2}{|c|}{ Composition (mg/kg) } \\
\hline & & /dry weight & /wet weight \\
\hline Choline & 7.085 & 181.122 & 18.022 \\
\hline Trigonelline & 7.642 & 324.271 & 32.265 \\
\hline Angustifoline & 8.073 & 2307.790 & 229.625 \\
\hline Sparteine & 9.035 & 16.494 & 1.641 \\
\hline Ellipcine & 9.785 & 38.235 & 3.804 \\
\hline Lupanine & 11.178 & 2372.643 & 236.078 \\
\hline 13- $\alpha$-Hydrorhombifoline & 11.296 & 51.108 & 5.085 \\
\hline 9-Octadecenamide & 12.810 & 40.003 & 3.980 \\
\hline Dihydro-oxo-demethoxyhaemanthamine & 14.126 & 40.477 & 4.028 \\
\hline Augustamine & 14.915 & 1948.528 & 193.879 \\
\hline Oxoassoanine & 15.375 & 331.419 & 32.976 \\
\hline Cinchonidine & 16.646 & 335.341 & 33.366 \\
\hline Cinchonine & 16.347 & 144.085 & 14.337 \\
\hline Crinane- $3 \alpha$-ol & 16.428 & 182.460 & 18.155 \\
\hline Buphanidrine & 16.544 & 28.978 & 2.883 \\
\hline Indicine-N-oxide & 17.472 & 119.855 & 11.926 \\
\hline Powelline & 18.669 & 41.741 & 4.153 \\
\hline Undulatine & 18.763 & 26.893 & 2.676 \\
\hline Ambelline & 19.669 & 41.741 & 4.153 \\
\hline 6-Hydroxybuphanidrine & 20.552 & 20.539 & 2.044 \\
\hline Acronycine & 21.239 & 43.732 & 4.351 \\
\hline Monocrotaline & 21.388 & 95.111 & 9.464 \\
\hline 6-Hydroxypowelline & 21.734 & 65.350 & 6.502 \\
\hline Nitidine & 22.347 & 80.852 & 8.045 \\
\hline Crinamidine & 23.960 & 562.134 & 55.932 \\
\hline $1 \beta, 2 \beta$-Epoxyambelline & 24.729 & 30.717 & 3.056 \\
\hline 6-Hydroxyundulatine & 24.842 & 40.889 & 4.069 \\
\hline Epoxy-3,7-dimethoxycrinane-11-one & 25.615 & 7.0315 & 0.700 \\
\hline Akuammidine & 26.919 & $70,420.759$ & 7006.866 \\
\hline Echitamidine & 26.786 & 488.511 & 48.607 \\
\hline Voacangine & 27.072 & $16,907.728$ & 1682.319 \\
\hline Mitraphylin & 27.624 & 0.010 & 0.001 \\
\hline Camptothecin & 28.229 & 5.434 & 0.541 \\
\hline Echitamine & 28.451 & 5063.374 & 503.806 \\
\hline Colchicine & 29.036 & 1.322 & 0.132 \\
\hline Emetine & 29.462 & 8.172 & 0.813 \\
\hline Tetrandrine & 30.153 & 1.786 & 0.178 \\
\hline Thalicarpin & 30.050 & 2.639 & 0.263 \\
\hline Paclitaxel & 32.211 & 3.238 & 0.322 \\
\hline Total alkaloids & & $102,421.000$ & $10,190.890$ \\
\hline
\end{tabular}


Studies have shown that ellagic acid has anti-allergic, anticancer, anti-depressant, anti-diabetic, anti-inflammatory, antimalarial, antioxidant, anti-wrinkle, neuroprotective [50], anti-apoptotic, anti-atherogenic, anti-proliferative and chemo-preventive [51] activities. Silymarin is an anti-arthritic, anti-carcinogenic, anti-diabetic, anti-hypercholesterolemic, anti-inflammatory, anti-obesity, antioxidant, cardioprotective, gastroprotective, hepatoprotective, immunomodulatory, neuroprotective and neurotropic [52] agent.

Myricetin exhibits antibacterial, anti-gonadotropic, antioxidant [34], anti-carcinogenic, anti-diabetic, antihyperlipidemic, anti-inflammatory, antimicrobial, antioxidant, anti-platelet, antiviral and cytoprotective [53] activities. Biochanin A has been reported to have anticancer, anti-inflammatory, antimicrobial, antioxidant, immunomodulatory and hepatoprotective [54] activities. Robinetin is a chemopreventive [55] agent; while butein is an antioxidant, pro-apoptotic and hepatoprotective [56] agent.

Baicalein is a potent free radical scavenger and xanthine oxidase inhibitor, thus improving endothelial function and conferring cardiovascular protective actions against oxidative stress-induced cell injury. Baicalein has anti-arteriosclerotic, antibacterial, antihypertensive, anti-inflammatory, anti-mitogenic, antioxidant, anti-proliferative, anti-thrombotic, antitumor, antiviral and cardio-protective [57] activities. Quercetin has been reported to have anti-allergic, anti-arthritic, antibacterial, anti-carcinogenic, anti-cancer, anti-cataractogenic, anti-diabetic, antihypertensive, anti-inflammatory, antioxidant, antiviral, cardio-protective, gastro-protective, hepatoprotective and hypocholesterolemic [34] [58] activities.

The carotene and lutein contents of the leaves were higher than those of cabbage, but lower than those of amaranth [59]. Carotenoids have been widely accepted as safe chemicals for food supplementation and neutraceutical purposes due to their intense coloring abilities, their role as precursors of vitamin A, and their antioxidant activity in animals [34] [60]. They have been shown to be beneficial in preventing major health problems in developing and developed countries, including cancers, cardiovascular and coronary heart diseases, photosensitivity disorders, age-related macular degeneration and cataract [60]. Carotenes and lutein protect against uterine, prostate, breast, colorectal and lung cancers [34]. Carotenes, in addition, have pro-vitamin A and antioxidant [34] activities.

The leaves had higher caffeic, vanilic and 4-hydroxybenzoic acids, but lower ferulic acid contents than red cabbage [61]. Phenolic acids are powerful antioxidants and have been reported to demonstrate antibacterial, antiviral, anti-carcinogenic, anti-inflammatory and vasodilatory actions [61]. 4-Hydroxybenzaldehyde is used as a flavor and fragrance agent. Caffeic acid has anti-diabetic [50], antioxidant [62], anti-inflammatory and photo-protective [63] activities. 4-Hydroxybenzoic acid esters (also called parabens), are widely used as antimicrobial agents in a large variety of food, pharmaceutical, and cosmetic products due to their excellent antimicrobial activities, low toxicity and stability over a wide $\mathrm{pH}$ range [64]. 4-Hydroxybenzoic acid has antifungal, anti-mutagenic, anti-sickling, estrogenic and antimicrobial [62] activities. Vanillic acid (4-hydroxy-3-methoxybenzoic acid) is used as a flavoring agent. It has anti-sickling, anthelmintic, hepatoprotective, immune-modulating, anti-inflammatory, antifungal, anti-mutagenic, estrogenic and antimicrobial [62] [65] activities. It also inhibits snake venom 5'-nucleotidase [62] [65]. Ferulic acid has antioxidant, hypocholesterolemic, anti-inflammatory, photo-protective, cardio-protective and sperm viability boosting activities [62].

The leaves had higher total phytosterols, stigmasterol and sitosterol contents than those of white cabbage and onions [66]. Phytosterols have anti-inflammatory [34], hypocholesterolemic, anti-cancer [34] [66], anti-ulcer, anti-fungal, anti-atherogenic, antioxidant [67] and estrogenic [68] activities. Stigmasterol has anti-osteoarthritic, anti-hypercholestrolemic, cytotoxicity, antitumor, thyroid inhibiting, hypoglycemic, anti-mutagenic, antioxidant, anti-inflammatory, anticonvulsant and sedative [69], chemopreventive, anticancer, estrogenic [70] activities. $\beta$-Sitosterol has been reported to have anti-inflammatory, anti-neoplastic, immune-modulating, antipyretic [34], cholesterol-lowering, antipyretic, antineoplastic, anti-diabetic, cardio-protective, estrogenic [68], antimicrobial, antioxidant, chemopreventive, anticancer and antipyretic [71] activities. It is used in the treatment of benign prostate hyperplasia [71], and alters sex steroid levels [68]. Sitosterylglucoside has been identified as an "adaptogens" [67]. According to Berger and colleagues [67], adaptogens are a group of natural plant products which promote overall health without the rapid response normally elicited by a drug and without the side effects associated with any drug used.

The leaves had higher total tannin content than red pepper [72], but lower tannic acid content than cabbage [73]. Tannins have antioxidant, antimicrobial [34], anticancer [74] activities. Tannic acid has antihypertensive, anti-diarrheal, anti-cancer, anti-asthmatic, cardioprotective, anti-diabetic, anti-cataractogenic, tumor inhibition, anti-inflammatory, anti-adipogenic [74] and hepatoprotective [75] activities. 
The leaves had higher total lignans contents than garlic and lettuce [76]. Lignans have antioxidant, anticancer, anti-diabetic, antiviral, bactericidal, anti-inflammatory, anti-atherosclerotic, cardio-protective [77], anti-hepatotoxic, antitumor, antiplatelet aggregation [78] and estrogen-like [76] activities. Galgravin has been reported to exhibit anti-inflammatory, anti-malarial, anti-platelet, anti-resorptive [79], anti-rheumatoid, neurotrophic and neuroprotective [28] activities.

Akuammidine has antibacterial, antifungal [80], antimalarial, anti-inflammatory, hypotensive, skeletal muscle relaxant, local analgesic and anti-depressant [81] activities. Voacangine has been reported to have cardiovascular toning, central nervous system depressant, anti-convulsive, anti-pyretic, analgesic, local anesthetic, acetyl cholinesterase inhibitory [82], anti-angiogenic [83] and anti-mycobacterial [84] activities. Studies have shown that echitamine has diuretic, hypotensive [85], central nervous system depressant and antiepileptic [86] properties. Augustifoline is a bacteriostatic agent [87] while lupanine is an anti-arrhythmic, hypotensive, hypoglycemic, $\beta$-glucosidase inhibitory, bacteriostatic [87], oxytocic and central nervous system depressant [88] agent.

Finally, the present study showed that the leaves of Tridax procumbens contain a variety of biologically active phytochemicals. The beneficial roles of these bioactive phytochemical constituents can be harnessed in the diet, making them veritable tools for nutritional therapy. This, therefore, emphasizes the potential of these leaves as a functional food.

\section{References}

[1] Alissa, E.M. and Ferns, G.A. (2012) Functional Foods and Nutraceuticals in the Primary Prevention of Cardiovascular Diseases. Journal of Nutrition and Metabolism, 2012, Article ID: 569486. http://dx.doi.org/10.1155/2012/569486

[2] Cencic, A. and Chingwaru, W. (2010) The Role of Functional Foods, Nutraceuticals, and Food Supplements in Intestinal Health. Nutrients, 2, 611-625. http://dx.doi.org/10.3390/nu2060611

[3] Hasler, C.M. (1998) Functional foods: Their Role in Disease Prevention and Health Promotion. Food Technology, 52, 57-62.

http://www.ift.org/ /media/Knowledge\%20Center/Science\%20Reports/Scientific\%20Status\%20Summaries/funcfood 1198.pdf

[4] Hasler, C.M. (2002) Functional Foods: Benefits, Concerns and Challenges-A Position Paper from the American Council on Science and Health. Journal of Nutrition, 132, 3772-3781. http://jn.nutrition.org/content/132/12/3772.full.pdf+html

[5] Kumari, S., Jain, S., Pal, R., Nain, S. and Paliwal, S. (2013) Pharmacognostical, Phytochemical and Pharmacological Investigation on Leaves of Tridax procumbens Linn. International Journal of Pharmaceutical Sciences and Research, 4, 792-795.

http://www.researchgate.net/publication/259532847_37_Vol.44_Issue_2_Feb_2013_IJPSR_RA-2025_Paper_37

[6] Patel, N.S., Jain, D.K., Nagar, H., Patel, A. and Chandel, H.S. (2011) Evaluation of Analgesic and Antipyretic Activity of Tridax procumbens Leaves Extract. RGUHS Journal of Pharmaceutical Sciences, 1, 226-231. http://dx.doi.org/10.5530/rjps.2011.3.9

[7] Prajapati, K., Singh, D., Mishra, S.B., Dubey, P. and Sangameswaran, B. (2008) Pharmacognostical and Preliminary Phytochemical Studies of Leaves of Tridax procumbens L. Ethnobotanical Leaflets, 12, 1283-1289.

http://opensiuc.lib.siu.edu/cgi/viewcontent.cgi?article=1207andcontext=ebl

[8] Sharma, H.P. and Rawal, A.K. (2013) Health Security in Ethnic Communities through Nutraceutical Leafy Vegetables. Journal of Environmental Research and Development, 7, 1423-1429. http://www.jerad.org/ppapers/dnload.php?vl=7andis=4andst=1423

[9] Ikewuchi, C.C. and Ikewuchi, J.C. (2009) Comparative Study of the Vitamin Composition of Some Common Nigerian Medicinal Plants. Pacific Journal of Science and Technology, 10, 367-371. http://www.akamaiuniversity.us/PJST10_1_367.pdf

[10] Ikewuchi, J.C. and Ikewuchi, C.C. (2009) Comparative Study of the Mineral Elements Composition of Some Common Nigerian Medicinal Plants. Pacific Journal of Science and Technology, 10, 362-366. http://www.akamaiuniversity.us/PJST10_1_362.pdf

[11] Ikewuchi, J.C., Ikewuchi, C.C. and Igboh, N.M. (2009) Chemical Profile of Tridax procumbens Linn. Pakistan Journal of Nutrition, 8, 548-550. http://dx.doi.org/10.3923/pjn.2009.548.550

[12] Prabhu, V.V., Nalini, G., Chidambaranathan, N. and Kisan, N.S. (2011) Evaluation of Anti-Inflammatory and Analgesic Activity of Tridax procumbens Linn against Formalin, Acetic Acid and CFA Induced Pain Models. International Journal of Pharmacy and Pharmacological Science, 3, 126-130. http://www.ijppsjournal.com/Vol3Issue2/2021.pdf

[13] Ikewuchi, J.C. and Ikewuchi, C.C. (2013) Moderation of Haematological Indices, Plasma Electrolytes and Markers of 
Hepato-Renal Function in Sub-Chronic Salt-Loaded Rats by an Aqueous Leaf Extract of Tridax procumbens Linn (Asteraceae). Pacific Journal of Science and Technology, 14, 362-369. http://www.akamaiuniversity.us/PJST14_1_362.pdf

[14] Petchi, R.R., Vijaya, C. and Parasuraman, S. (2013) Anti-Arthritic Activity of Ethanolic Extract of Tridax procumbens (Linn.) in Sprague Dawley Rats. Pharmacognosy Research, 5, 113-117. http://dx.doi.org/10.4103/0974-8490.110541

[15] Bhagwat, D.A., Suresh, K.G. and Rahul, A.S. (2008) Anti-Diabetic Activity of Leaf Extract of Tridax procumbens. International Journal of Green Pharmacy, 2, 126-128. http://www.greenpharmacy.info/text.asp?2008/2/2/126/41188 http://dx.doi.org/10.4103/0973-8258.41188

[16] Ikewuchi, J.C. (2011) Alteration of Plasma Biochemical, Haematological and Ocular Oxidative Indices of Alloxan Induced Diabetic Rats by Aqueous Extract of Tridax procumbens Linn (Asteraceae). EXCLI Journal, 11, 291-308. http://hdl.handle.net/2003/29680

[17] Pareek, H., Sharma, S., Khajja, B.S., Jain, K. and Jain, G.C. (2008) Evaluation of Hypoglycemic and Anti-Hyperglycemic Potential of Tridax procumbens (Linn.). BMC Complementary and Alternative Medicine, 9, 48. http://dx.doi.org/10.1186/1472-6882-9-48

[18] Ikewuchi, J.C., Onyeike, E.N., Uwakwe, A.A. and Ikewuchi, C.C. (2011) Effect of Aqueous Extract of the Leaves of Tridax procumbens Linn on Blood Pressure Components and Pulse Rates of Sub Chronic Salt-Loaded Rats. Pacific Journal of Science and Technology, 12, 381-389. http://www.akamaiuniversity.us/PJST12_1_381.pdf

[19] Salahdeen, H.M., Yemitan, O.K. and Alada, A.R.A. (2004) Effect of Aqueous Leaf Extract of Tridax procumbens on Blood Pressure and Heart Rate in Rats. African Journal Biomedical Research, 7, 27-29.

[20] Ravikumar, V., Shivashangari, K.S. and Devaki, T. (2005) Hepatoprotective Activity of Tridax procumbens against DGalactosamine/Lipopolysaccharide-Induced Hepatitis in Rats. Journal of Ethnopharmacology, 101, 55-60. http://dx.doi.org/10.1016/j.jep.2005.03.019

[21] Yoga Latha Jr., L., Darah, I., Sasidharan, S. and Jain, K. (2009) Antimicrobial Activity of Emilia sonchifolia DC., Tridax procumbens L. and Vernonia cinerea L. of Asteracea Family: Potential as Food Preservatives. Malaysian Journal of Nutrition, 15, 223-231. http://nutriweb.org.my/publications/mjn0015_2/Sasidharan.pdf

[22] Ikewuchi, J.C. (2012) An Aqueous Extract of the Leaves of Tridax procumbens Linn (Asteraceae) Protected against Carbon Tetrachloride Induced Liver Injury in Wistar Rats. Pacific Journal of Science and Technology, 13, 519-527. http://www.akamaiuniversity.us/PJST13_1_519.pdf

[23] Ikewuchi, J.C., Onyeike, E.N., Uwakwe, A.A. and Ikewuchi, C.C. (2011b) Weight Reducing and Hypocholesterolemic Effects of Aqueous Extract of the Leaves of Tridax procumbens Linn on Sub-Chronic Salt-Loaded Rats. International Journal of Biological and Chemical Sciences, 5, 680-687.

[24] Tram, N.T.C., Mitova, M., Bankova, V., Handjieva, N. and Popov, S.S. (2002) GC-MS of Crinum latifolium L. Alkaloids. Zeitschrift für Naturforschung, 57c, 239-242. http://www.znaturforsch.com/ac/v57c/s57c0239.pdf

[25] Rodriguez-Amaya, D.B. and Kimura, M. (2004) HarvestPlus Handbook for Carotenoid Analysis. HarvestPlus Technical Monograph 2, International Food Policy Research Institute (IFPRI) and International Center for Tropical Agriculture (CIAT), Washington DC and Cali. http://www.ifpri.org/sites/default/files/publications/hptech02.pdf

[26] Millogo-Kone, H., Lompo, M., Kini, F., Asimi, S., Guissou, I.P. and Nacoulma, O. (2009) Evaluation of Flavonoids and Total Phenolic Contents of Stem Bark and Leaves of Parkia biglobosa (Jacq.) Benth. (Mimosaceae)-Free Radical Scavenging and Antimicrobial Activities. Research Journal of Medical Science, 3, 70-74. http://medwelljournals.com/abstract/?doi=rjmsci.2009.70.74

[27] Ortan, A., Popescu, M.-L., Gaita, A.-L., Dinu-Pîrvu, C. and Câmpeanu, G.H. (2009) Contributions to the Pharmacognostical Study on Anethum graveolens, Dill (Apiaceae). Romanian Biotechnology Letters, 14, 4342-4348. http://www.rombio.eu/rbl2vol14/cnt/Lucr-18.pdf

[28] Chapman, J.M., Knoy, C., Kindscher, K., Brown, R.C.D. and Niemann, S. (2006) Identification of Antineoplastic and Neurotrophic Lignans in Medicinal Prairie Plants by Liquid Chromatography Electron Impact Mass Spectrometry (LC/EI/MS). Life Sciences. http://dobyobktvl6u3.cloudfront.net/media/filer_private/2012/01/06/kclifescience2006_1.pdf

[29] Ndoumou, D.O., Ndzomo, G.T. and Djocgoue, P.F. (1996) Changes in Carbohydrate, Amino Acid and Phenol Contents in Cocoa Pods from Three Clones after Infection with Phytophthora megakarya Bra. and Grif. Annals of Botany, 77, 153-158. http://dx.doi.org/10.1006/anbo.1996.0017

[30] AOAC International (Horwitiz, W., Ed.) (2006) Official Methods of Analysis of the AOAC. 18th Edition, AOAC International, Washington DC.

[31] Luthar, Z. (1992) Polyphenol Classification and Tannin Content of Buckwheat Seeds (Fagopyrum esculentum Moench). Fagopyrum, 12, 36-42. http://fagopyrum.uhostall.com/Fagopyrum/Fagopyrum/Each/Fag(12)/Fag(12)-36.pdf

[32] Health Canada (1999) Determination of Alkaloids in Whole Tobacco. Health Canada-Official Method T-301. To- 
bacco Control Programme, Ottawa, Canada K1A 0K9.

[33] Harnly, J.M., Doherty, R.F., Beecher, G.R., Holden, J.M., Haytowitz, D.B., Bhagwat, S., et al. (2006) Flavonoid Content of U.S. Fruits, Vegetables, and Nuts. Journal of Agriculture and Food Chemistry, 54, 9966-9977. http://dx.doi.org/10.1021/jf061478a

[34] Dillard, C.J. and German, J.B. (2000) Phytochemicals: Nutraceuticals and Human Health. Journal of the Science of Food and Agriculture, 80, 1744-1756. http://dx.doi.org/10.1002/1097-0010(20000915)80:12<1744::AID-JSFA725>3.0.CO;2-W

[35] López-Lázaro, M. (2009) Distribution and Biological Activities of the Flavonoid Luteolin. Mini-Reviews in Medicinal Chemistry, 9, 31-59. http://dx.doi.org/10.2174/138955709787001712

[36] Calderón-Montaño, J.M., Burgos-Morón, E., Pérez-Guerrero, C. and López-Lázaro, M. (2011) A Review on the Dietary Flavonoid Kaempferol. Mini-Reviews in Medicinal Chemistry, 11, 298-344. http://dx.doi.org/10.2174/138955711795305335

[37] Ahmad, M., Gilani, A.-U.-H., Aftab, K. and Ahmad, V.U. (1993) Effects of Kaempferol-3-O-Rutinoside on Rat Blood Pressure. Phytotherapy Research, 7, 314-316. http://dx.doi.org/10.1002/ptr.2650070411

[38] Panda, S. and Kar, A. (2007) Apigenin (4’,5,7-Trihydroxyflavone) Regulates Hyperglycaemia, Thyroid Dysfunction and Lipid Peroxidation in Alloxan-Induced Diabetic Mice. Journal of Pharmacy and Pharmacology, 59, 1543-1548. http://dx.doi.org/10.1211/jpp.59.11.0012

[39] Saeed, M.K., Deng, Y., Perveen, Z., Ahmad, W., Dai, R. and Yu, Y. (2006) Optimal Recovery of Apigenin from Torreya grandis by Extraction, Fractionation and Structure Elucidation. Proceedings of the 2006 WSEAS International Conference on Cellular and Molecular Biology, Biophysics and Bioengineering, Athens, 14-16 July 2006, 32-38. http://www.wseas.us/e-library/conferences/2006athensbio/papers/536-141.pdf

[40] Norton, K.J. (2011) Phytochemicals-11 Health Benefits of Naringenin. Health Articles. http://kylenorton.healthblogs.org/2011/12/16/phytochemicals-11-health-benefits-of-naringenin/

[41] Su, W.-W., Wang, Y.-G., Fang, T.-Z., Peng, W. and Wu, Z. (2009) Uses of Naringenin, Naringin and Salts Thereof as Expectorants in the Treatment of Cough, and Compositions Thereof. European Patent Specification, EP1591123B1. http://www.freepatentsonline.com/EP1591123.pdf

[42] Sutherland, B.A., Rahman, R.M.A. and Appleton, I. (2006) Mechanisms of Action of Green Tea Catechins, with a Focus on Ischemia-Induced Neurodegeneration. Journal of Nutritional Biochemistry, 17, 291-306. http://dx.doi.org/10.1016/j.jnutbio.2005.10.005

[43] Ghayur, M.N., Khan, H. and Gilani, A.H. (2007) Antispasmodic, Bronchodilator and Vasodilator Activities of (+)Catechin, a Naturally Occurring Flavonoid. Archives of Pharmacal Research, 30, 970-975. http://dx.doi.org/10.1007/BF02993965

[44] Chakravarthy, B.K., Gupta, S. and Gode, K.D. (1982) Functional B-Cells Regeneration in the Islets of Pancreas in Alloxan-Induced Diabetic Rats by (-)-Epicatechin. Life Sciences, 31, 2693-2697. http://dx.doi.org/10.1016/0024-3205(82)90713-5

[45] Houston, M.C. (2005) Nutraceuticals, Vitamins, Antioxidants, and Minerals in the Prevention and Treatment of Hypertension. Progress in Cardiovascular Diseases, 47, 396-449. http://dx.doi.org/10.1016/j.pcad.2005.01.004

[46] Kim, M.J. and Lim, Y. (2013) Protective Effect of Short-Term Genistein Supplementation on the Early Stage in Diabetes-Induced Renal Damage. Mediators of Inflammation, 2013, Article ID: 510212.

[47] Ikemura, M., Sasaki, Y., Giddings, J.C. and Yamamoto, J. (2012) Protective Effects of Nobiletin on Hypertension and Cerebral Thrombosis in Stroke-Prone Spontaneously Hypertensive Rats (SHRSP). Food and Nutrition Sciences, 3, 1539-1546. http://dx.doi.org/10.4236/fns.2012.311201

[48] Lam, K.H., Alex, D., Lam, I.K., Tsui, S.K.W., Yang, Z.F. and Lee, S.M.Y. (2011) Nobiletin, a Polymethoxylated Flavonoid from Citrus, Shows Anti-Angiogenic Activity in a Zebrafish in Vivo Model and HUVEC in Vitro Model. Journal of Cellular Biochemistry, 112, 3313-3321. http://dx.doi.org/10.1002/jcb.23257

[49] Luque-Alcaraz, A.G., Lizardi, J., Goycoolea, F.M., Valdez, M.A., Acosta, A.L., Iloki-Assanga, S.B., et al. (2012) Characterization and Antiproliferative Activity of Nobiletin-Loaded Chitosan Nanoparticles. Journal of Nanomaterials, 2012, Article ID: 265161.

[50] Dhingra, D. and Chhillar, R. (2012) Antidepressant-Like Activity of Ellagic Acid in Unstressed and Acute Immobilization-Induced Stressed Mice. Pharmacological Reports, 64, 796-807. http://www.if-pan.krakow.pl/pjp/pdf/2012/4_796.pdf http://dx.doi.org/10.1016/S1734-1140(12)70875-7

[51] Özkaya, A., Çiftçi, H., Dayangaç, A., Çevrimli, B.S., Ölçücü, A. and Çelik, S. (2013) Effects of Ellagic Acid and Hesperetin on Levels of Some Elements in Livers of Aluminum-Induced Rats. Turkish Journal of Biochemistry, 38, 345-349. http://dx.doi.org/10.5505/tjb.2013.29291 
[52] Dixit, N., Baboota, S., Kohli, K., Ahmad, S. and Ali, J. (2007) Silymarin: A Review of Pharmacological Aspects and Bioavailability Enhancement Approaches. Indian Journal of Pharmacology, 39, 172-179. http://dx.doi.org/10.4103/0253-7613.36534

[53] Li, Y. and Ding, Y. (2012) Minireview: Therapeutic Potential of Myricetin in Diabetes Mellitus. Food Science and Human Wellness, 1, 19-25. http://dx.doi.org/10.1016/j.fshw.2012.08.002

[54] Breikaa, R.M., Algandaby, M.M., El-Demerdash, E. and Abdel-Naim, A.B. (2013) Biochanin A Protects against Acute Carbon Tetrachloride-Induced Hepatotoxicity in Rats. Bioscience, Biotechnology and Biochemistry, 77, 909-916. http://dx.doi.org/10.1271/bbb.120675

[55] Middleton Jr., E., Kandaswami, C. and Theoharides, T.C. (2000) The Effects of Plant Flavonoids on Mammalian Cells: Implications for Inflammation, Heart Disease, and Cancer. Pharmacological Reviews, 52, 673-751. http://pharmrev.aspetjournals.org/content/52/4/673.full

[56] Sehrawat, A. and Kumar, V. (2012) Butein Imparts Free Radical Scavenging, Anti-Oxidative and Proapoptotic Properties in the Flower Extracts of Butea monosperma. Biocell, 36, 63-71.

[57] Huang, Y., Tsang, S.-Y., Yao, X. and Chen, Z.-Y. (2013) Biological Properties of Baicalein in Cardiovascular System. Current Drug Target-Cardiovascular and Hematological Disorders, 5, 177-184. http://dx.doi.org/10.2174/1568006043586206

[58] Lakhanpal, P. and Rai, D.K. (2007) Quercetin: A Versatile Flavonoid. Internet Journal of Medical Update, 2, $22-37$. http://www.akspublication.com/Paper05Jul-Dec2007.pdf

[59] Kidmose, U., Yang, R.-Y., Thilsted, S.H., Christensen, L.P. and Brandt, K. (2006) Content of Carotenoids in Commonly Consumed Asian Vegetables and Stability and Extractability during Frying. Journal of Food Composition and Analysis, 19, 562-571. http://dx.doi.org/10.1016/j.jfca.2006.01.011

[60] Mayne, S.T. (1996) Beta-Carotene, Carotenoids, and Disease Prevention in Humans. FASEB Journal, 10, 690-701. http://www.fasebj.org/content/10/7/690.full.pdf\#page=1andview=FitH

[61] Mattila, P. and Hellstrom, J. (2006) Phenolic Acids in Potatoes, Vegetables, and Some of Their Products. http://www.researchgate.net/publication/222004840_Phenolic_acids_in_potatoes_vegetables_and_some_of_their_prod ucts/file/9fcfd5063dac6b10bd.pdf

[62] Oksana, S., Marian, B., Mahendra, R. and Bo, S.H. (2012) Plant Phenolic Compounds for Food, Pharmaceutical and Cosmetics Production. Journal of Medicinal Plants Research, 6, 2526-2539.

[63] Gugliucci, A., Bastos, D.H.M., Schulze, J. and Souza, M.F.F. (2009) Caffeic and Chlorogenic Acids in Ilex paraguariensis Extracts Are the Main Inhibitors of AGE Generation by Methylglyoxal in Model Proteins. Fitoterapia, 80, 339-344. http://dx.doi.org/10.1016/j.fitote.2009.04.007

[64] Valkova, N., Lépine, F., Valeanu, L., Dupont, M., Labrie. L., Bisaillon, J.G., et al. (2001) Hydrolysis of 4-Hydroxybenzoic Acid Esters (Parabens) and Their Aerobic Transformation into Phenol by the Resistant Enterobacter cloacae Strain EM. Applied and Environmental Microbiology, 67, 2404-2409. http://dx.doi.org/10.1128/AEM.67.6.2404-2409.2001

[65] Khadem, S. and Marles, R.J. (2010) Monocyclic Phenolic Acids; Hydroxy- and Polyhydroxybenzoic Acids: Occurrence and Recent Bioactivity Studies. Molecules, 15, 7985-8005. http://dx.doi.org/10.3390/molecules15117985

[66] Piironen, V., Toivo, J., Puupponen-Pimiä, R. and Lampi, A.-M. (2003) Plant Sterols in Vegetables, Fruits and Berries. Journal of the Science of Food and Agriculture, 83, 330-337. http://dx.doi.org/10.1002/jsfa.1316

[67] Berger, A., Jones, P.J.H. and Abumweis, S.S. (2004) Plant Sterols: Factors Affecting Their Efficacy and Safety as Functional Food Ingredients. Lipids in Health and Diseases, 3, 5. http://dx.doi.org/10.1186/1476-511X-3-5

[68] Ju, Y.H., Clausen, L.M., Allred, K.F., Almada, A.L. and Helferich, W.G. (2004) $\beta$-Sitosterol, $\beta$-sitosterol Glucoside, and a Mixture of $\beta$-Sitosterol and $\beta$-Sitosterol Glucoside Modulate the Growth of Estrogen-Responsive Breast Cancer Cells in Vitro and in Ovariectomized Athymic Mice. Journal of Nutrition, 134, 1145-1151. http://jn.nutrition.org/content/134/5/1145.long\#fn-1

[69] Kaur, N., Chaudhary, J., Jain, A. and Kishore, L. (2011) Stigmasterol: A Comprehensive Review. International Journal of Pharmaceutical Sciences and Research, 2, 2259-2265. http://ijpsr.com/?action=download_pdf\&postid=18101

[70] Boldrin, P.K., Resende, F.A., Höhne, A.P.O., de Camargo, M.S., Espanha, L.G., Catarine Nogueira, H., et al. (2013) Estrogenic and Mutagenic Activities of Crotalaria pallida Measured by Recombinant Yeast Assay and Ames Test. BMC Complementary and Alternative Medicine, 13, 216. http://dx.doi.org/10.1186/1472-6882-13-216

[71] Sen, A., Dhavan, P., Shukla, K.K., Singh, S. and Tejovathi, G. (2012) Analysis of IR, NMR and Antimicrobial Activity of $\beta$-Sitosterol Isolated from Momordica charantia. Science Secure Journal of Biotechnology, 1, 9-13. http://www.researchgate.net/publication/235995077 Analysis of IR NMR and Antimicrobial Activity of -Sitoster ol_Isolated_from_Momordica_charantia/file/3deec515591cbc2a35.pdf 
[72] Atanassova, M. and Christova-Bagdassarian, V. (2009) Determination of Tannins Content by Titrimetric Method for Comparison of Different Plant Species. Journal of the University of Chemical Technology and Metallurgy, 44, 413-415. http://dl.uctm.edu/journal/node/j2009-4/14_Maria_Atanasova_413-416.pdf

[73] Mosha, T.C., Gaga, H.E., Pace, R.D., Laswai, H.S. and Mtebe, K. (1995) Effect of Blanching on the Content of Antinutritional Factors in Selected Vegetables. Plant Foods for Human Nutrition, 47, 361-367. http://dx.doi.org/10.1007/BF01088275

[74] Namkung, W., Thiagarajah, J.R., Phuan, P.-W. and Verkman, A.S. (2010) Inhibition of $\mathrm{Ca}^{2+}$-Activated $\mathrm{Cl}^{-} \mathrm{Channels}^{-}$ by Gallotannins as a Possible Molecular Basis for Health Benefits of Red Wine and Green Tea. FASEB Journal, 24, 4178-4186. http://dx.doi.org/10.1096/fj.10-160648

[75] Mittal, D.K., Joshi, D. and Shukla, S. (2010) Protective Effects of Polygonum bistorta (Linn.) and Its Active Principle against Acetaminophen-Induced Toxicity in Rats. Asian Journal of Experimental Biology and Science, 1, 951-958. http://www.researchgate.net/profile/Deepmala_Joshi2/publication/261298467_Protective_effect_of_Polygonum_bistor ta_Linn._and_its_active_principle_against_acetaminophen-induced_toxicity_in_rats/links/00b7d533d4d9d511ba00000 $\underline{0 . p d f ? i n V i e w e r=t r u e \& d i s a b l e C o v e r P a g e=t r u e \& o r i g i n=p u b l i c a t i o n \_d e t a i l ~}$

[76] Thompson, L.U., Boucher, B.A., Liu, Z., Cotterchio, M. and Kreiger, N. (2006) Phytoestrogen Content of Foods Consumed in Canada, Including Isoflavones, Lignans, and Coumestan. Nutrition and Cancer, 54, 184-201.

http://www.encognitive.com/files/Phytoestrogen\%20Content\%20of\%20Foods\%20Consumed\%20in\%20Canada,\%20In cluding\%20Isoflavones, \%20Lignans,\%20and\%20Coumestan.pdf http://dx.doi.org/10.1207/s15327914nc5402_5

[77] Zanwar, A.A., Hegde, M.V. and Bodhankar, S.L. (2011) Cardioprotective Activity of Flax Lignin Concentrate Extracted from Seeds of Linum usitatissimum in Isoprenalin Induced Myocardial Necrosis in Rats. Interdisciplinary Toxicology, 4, 90-97. http://dx.doi.org/10.2478/v10102-011-0016-8

[78] Kim, M.-G., Lee, C.-H. and Lee, H.-S. (2010) Anti-Platelet Aggregation Activity of Lignans Isolated from Schisandra chinensis Fruits. Journal of Korean Society of Applied Biology and Chemistry, 53, 740-745. http://dx.doi.org/10.3839/jksabc.2010.112

[79] Asai, M., Lee, J.-W., Itakura, Y., Choi, B.-K., Yonezawa, T., Teruya, T., et al. (2012) Effects of Veraguensin and Galgravin on Osteoclast Differentiation and Function. Cytotechnology, 64, 315-322. http://dx.doi.org/10.1007/s10616-011-9416-z

[80] Pawar, H.A., Shenoy, A.V., Narawade, P.D., Soni, P.Y., Shanbhag, P.P. and Rajal, V.A. (2011) Preservatives from Nature: A Review. International Journal of Pharmaceutical and Phytopharmacological Research, 1, 78-88. http://www.eijppr.com/6.Preservatives\%20from\%20Nature\%20A\%20Review.pdf

[81] Nyunaï, N. and Njifutié, N. (2006) Picralima nitida (Stapf) T. Durand and H. Durand. [Internet] Record from PROTA4U. Schmelzer, G.H. and Gurib-Fakim, A. (Editors). PROTA (Plant Resources of Tropical Africa/Ressources végétales de l'Afrique tropicale), Wageningen. http://www.prota4u.org/search.asp

[82] Pratchayasakul, W., Pongchaidecha, A., Chattipakorn, N. and Chattipakorn, S. (2008) Ethnobotany and Ethnopharmacology of Tabernaemontana divaricate. Indian Journal of Medical Research, 127, 317-335. http://icmr.nic.in/ijmr/2008/april/0405.pdf

[83] Kim, Y., Jung, H.J. and Kwon, H.J. (2012) A Natural Small Molecule Voacangine Inhibits Angiogenesis Both in vitro and in vivo. Biochemical and Biophysical Research Communication, 417, 330-334. http://dx.doi.org/10.1016/j.bbrc.2011.11.109

[84] Rastogi, N., Abaul, J., Goh, K.S., Devallois, A., Philogène, E. and Bourgeois, P. (1998) Antimycobacterial Activity of Chemically Defined Natural Substances from the Caribbean Flora in Guadeloupe. FEMS Immunology and Medical Microbiology, 20, 267-273. http://dx.doi.org/10.1111/j.1574-695X.1998.tb01136.x

[85] Ojewole, J.A.O. (1984) Studies on the Pharmacology of Echitamine, an Alkaloid from the Stem Bark of Alstonia boonei L. (Apocynaceae). Pharmaceutical Biology, 22, 121-143. http://dx.doi.org/10.3109/13880208409070663

[86] Nsour, W.M., Lau, C.B.-S. and Wong, I.C.K. (2000) Review on Phytotherapy in Epilepsy. Seizure, 9, 96-107. http://dx.doi.org/10.1053/seiz.1999.0378

[87] Sagen, A.-L. (2002) Phytochemical and Biological Investigations on Clathrotropis glaucophylla (Fabaceae), an Ingredient of Yanomamï Curare, Emphasizing on Quinolizidine Alkaloids. A Dissertation Submitted for the Degree of Doctor of Natural Sciences, to the Swiss Federal Institute of Technology, Zurich. Diss. ETH No. 14785. http://e-collection.library.ethz.ch/eserv/eth:25885/eth-25885-02.pdf

[88] Wink, M. (1997) Quinolizidine Alkaloids: Biological Activities and Potential Applications. In: Bardocz, S., Muzquiz, M. and Pusztai, A., Eds., Cost 98: Effects of Antinutrients on the Nutritional Value of Legume Diets, Vol. 4, Proceedings of the 4th Scientific Workshop, Madrid, 19-21 September 1996, European Commission, Directorate-General XII, Science, Research and Development, Luxembourg, 1-7. 